\title{
Successful Anaesthetic Management of Caesarean Section: An Experience on a Patient with Myasthenia Gravis and Difficult Airway
}

\author{
Akwasi Antwi-Kusi1 ${ }^{*}$, William Addison1, Elizabeth Esi Crentsil2, Anthony Yeboah ${ }^{2}$, \\ Rex Mawuli Kwadjo Djokoto ${ }^{2}$ \\ ${ }^{1}$ Department of Anaesthesiology, Komfo Anokye Teaching Hospital, Kumasi, Ghana \\ ${ }^{2}$ Department of Obstetrics and Gynaecology, Komfo Anokye Teaching Hospital, Kumasi, Ghana \\ Email: ^antwikusi@yahoo.com
}

How to cite this paper: Antwi-Kusi, A., Addison, W., Crentsil, E.E., Yeboah, A. and Djokoto, R.M.K. (2019) Successful Anaesthetic Management of Caesarean Section: An Experience on a Patient with Myasthenia Gravis and Difficult Airway. Open Journal of Obstetrics and Gynecology, 9, 789-793.

https://doi.org/10.4236/ojog.2019.96078

Received: May 3, 2019

Accepted: May 30, 2019

Published: June 3, 2019

Copyright $\odot 2019$ by author(s) and Scientific Research Publishing Inc. This work is licensed under the Creative Commons Attribution International License (CC BY 4.0).

http://creativecommons.org/licenses/by/4.0/

\begin{abstract}
The anaesthetic management of the pregnant woman with myasthenia gravis (MG) is very challenging to the anaesthesiologist. This situation becomes exaggerated in emergency settings in a low resource area, especially when attending anesthesiologists for the first time took care of her. Here, we present a case that illustrates this situation. A G3 P2 pregnant woman presented for an emergency caesarean section (CS) due to premature rupture of the membranes. The patient had been misinformed by her neurophysician not to undergo general anaesthesia at CS. She had no motor weakness and no bulbar symtoms. The only abnormal finding was a stiff neck and a Mallampati \#2 score, which predicted a difficult airway. Spinal anaesthesia was not successful after several attempts. General anaesthesia was considered but again intubation was not successful. We performed anesthesia with a second generation laryngeal mask airway with successful CS. Pregnant women with MG require personalised care from a multidisciplinary team. While regional anaesthesia is reported to be the better choice in CS for women with MG, regional anesthesia is not always possible. Anesthesia should be chosen in a patient-by-patient manner. The present case illustrates such conditions, of which description may be useful for anesthesiologists and obstetricians.
\end{abstract}

\section{Keywords}

Myasthenia Gravis, Failed Spinal, Difficult Intubation, Second Generation Laryngeal Mask Airway

\section{Introduction}

Myasthenia Gravis (MG) is a chronic autoimmune disease involving the neuro- 
muscular junction. The hallmark of the disease is weakness and rapid fatigability of voluntary skeletal muscles with repetitive use, followed by partial recovery with rest [1]. The incidence is about 1 in every 20,000 adults. It is twice as common in women as in men, and frequently affects young women in the second and third decades of life, which overlaps with the childbearing age [2] [3]. Here, we present a case of pregnant woman with MG, in whom anesthesia for caesarean section (CS) required special attention.

\section{Case Presentation}

A 38-year-old gravida-3 para-2 (vaginal and CS) patient was diagnosed as having MG 2 years ago. The patient presented for emergency CS due to premature rupture of the membranes. When the neurologist caring for the patient was contacted, he confirmed the diagnosis and informed us of the patient's medications. These included pyridostigmine $500 \mathrm{mg} b d$, tab prednisolone $10 \mathrm{mg}$ daily and tab mycophenslate $500 \mathrm{mg}$ bd. The patient's condition had been stable throughout her pregnancy, and the obstetrician was hoping for a normal spontaneous vaginal delivery when she developed premature rupture of membranes.

On admission, she had no muscle weakness and no bulbar symptoms such as dysphagia, dysarthria or nasal speech. She had no shortness of breath or dyspnoea. Airway examination revealed limited neck flexion and Mallampatti II classification. Her last meal intake was the previous night meaning the patient had fasted for more than 8 hours.

Before we discussed anaesthesia options with the patient, the patient objected to general anaesthesia on the advice of her neurologist, who had told her she would not wake up if she would undergo general anaesthesia. Our first choice of anaesthesia was neuraxial anaesthesia. Baseline preoperative monitoring revealed blood pressure 130/90 $\mathrm{mmHg}$, sinus rhythm of $94 \mathrm{bpm}$, and $\mathrm{SPO}_{2}$ of $96 \%$ on room air. From L3/4, the spinal block was attempted by an experienced anaesthesiologist; however, it was unsuccessful. After many attempts over almost an hour, spinal anaesthesia was abandoned. Our next option was to resort to general anaesthesia. Since the patient had objected to general anaesthesia on the advice of her neurologist, we explained to her that general anaesthesia was not contraindicated in MG patients. This case was especially challenging because it was an emergency: we could not postpone CS.

The patient was positioned supine with a left lateral tilt. Initial monitoring was normal with a BP of 130/80 $\mathrm{mmHg}$, pulse $105 \mathrm{bpm}$ and oxygen saturation of $98 \%$ on room air. Preoxygenation was done with $10 \mathrm{l} / \mathrm{min}$ of oxygen followed by $200 \mathrm{mg}$ of propofol. A test of mask ventilation was performed, after which laryngoscopy was tried without muscle relaxation. The patient was fully unconscious after the $200 \mathrm{mg}$ of propofol, chest expansion was good on test ventilation and the patient was well-relaxed. An attempt at laryngoscopy revealed a Cormack Lehane \#3 glottic view with very limited neck extension. A video-laryngoscope, glidescope or fibreoptic bronchoscope was not available. 
After a careful assessment, we concluded that endotracheal intubation would be extremely difficult since a video laryngoscope or other adjuncts were unavailable. With a very limited chance of successful intubation, a decision was made to not give any muscle relaxant. Instead, a laryngeal mask airway was inserted successfully to provide good ventilation. Anaesthesia was maintained with isoflurane $2 \%$ - 3\% MAC. The CS was successfully completed, lasting 40 minutes with no problems intraoperatively. The baby was delivered healthy with an APGAR score of 9/9 (1/5 minutes). There was no sign of muscle weakness and no bulbar symptoms.

\section{Discussion}

We present a pregnant woman with MG requiring emergent CS, who had an unsuspected difficult airway. She had a failed lumbar puncture and was also difficult to intubate. Eventually, general anesthesia with laryngeal mask airway was performed, with successfull anaesthesia and CS.

The basic defect resulting in skeletal muscle weakness and easy fatigability in MG is a decrease in the number of available receptors for acetylcholine at the postsynaptic neuromuscular junction. This decrease is due to their inactivation or destruction by circulating antibodies. Attachment of these antibodies to receptors for acetylcholine either blocks neurotransmitter access to receptors or accelerates degradation of receptors [4] [5]. Principles for anaesthesia management of patients with MG includes avoidance of neuromuscular blocking agents whenever possible. If necessary, reversal of the non-depolarizing neuromuscular blocking agent should be done with sugammadex instead of neostigmine whenever possible. Premedication should also be avoided, and the patient should rather be counselled and reassured. If premedication with midazolam must be used, it should be given in small incremental doses with close attention to signs of bulbar weakness and respiratory compromise [6].

The patient's fear was heightened by the fact that her neurologist had advised her not to receive general anaesthesia for CS. The neurologist should have referred the patient to an anaesthesiologist to discuss the choice of anaesthesia. The concern is with the use of muscle relaxation and not general anaesthesia. With MG, if muscle relaxants are needed, the dose of muscle relaxants should be reduced [7]. General anaesthesia is indeed recommended for CS in MG patients when they had significant bulbar or respiratory involvement to better control airway, oxygenation and secretions [8] [9]. There is, however, a possibility of a post-operative myasthenia crisis when general anaesthesia is used with muscle relaxants. This post-operative MG crisis can be predicted if the patient presents with the following: 1) vital capacity $<2$ to $2.9 ; 2$ ) duration of MG greater than six years; 3) history of chronic pulmonary disease; 4) preoperative bulbar symptoms; 5) history of myasthenia crisis; 6) intraoperative blood loss greater than $1000 \mathrm{mls}$ [10]. Fortunately, this crisis did not occur in this patient.

On airway examination, the patient had limited neck movement and a Mal- 
lampatti II score. We found no reports associating MG and stiff neck or difficult spinal anaesthesia. However, a Mallampatti score of II or more is an independent risk factor for a failed intubation [11].

For this patient, laryngoscopy was performed immediately after the administration of propofol and without administration of muscle relaxants. This provided an opportunity to assess the Comark-Lehane glottic score, which made us to decide the possible ease or difficulty of endotracheal intubation. In MG, neuromuscular blocking agents should be avoided whenever possible. These patients are resistant to depolarising neuromuscular blocking agents like succinylcholine therefore necessitating larger doses, though this is more prone to provoke a phase II block [12].

Propofol was used due to its rapid onset, short duration, and ability to quickly obtund respiratory reflexes. We decided not to attempt intubation but instead use an i-gel laryngeal mask airway (LMA), also described as a second generation LMA. This type of LMA has a second pot for suctioning the stomach. It is relatively safe when aspiration becomes a concern. A second-generation supraglottic airway device is recommended for use in the event of a failed intubation, as there is the potential for aspiration [13]. Recent obstetric difficult airway guidelines have emphasised preferentially managing oxygenation via alternative airway devices over repeated intubation attempts to secure the airway with an endotracheal tube [14] [15].

\section{Conclusion}

There are many challenges associated with managing the pregnant woman with MG who has to undergo CS. While regional anaesthesia is reported to be the better choice in CS for women with MG, regional anesthesia is not always possible. Anesthesia should be chosen in a patient-by-patient manner. The present case illustrates such conditions, of which description may be useful for anesthesiologists and obstetricians.

\section{Conflicts of Interest}

The authors declare no conflicts of interest regarding the publication of this paper.

\section{References}

[1] Almeida, C., Coutinho, E., Moreira, D., Santos, E. and Aguiar, J. (2010) Myasthenia Gravis and Pregnancy: Anaesthetic Management-A Series of Cases. European Journal of Anaesthesiology, 27, 985-990. https://doi.org/10.1097/EJA.0b013e32833e263f

[2] Pauche, W.C. (1983) Myasthenia Gravis. Clinical Obstetrics and Gynecology, 26, 592-604. https://doi.org/10.1097/00003081-198309000-00010

[3] Burke, M.E. (1993) Myasthenia Gravis and Pregnancy. The Journal of Perinatal \& Neonatal Nursing, 7, 11-21. https://doi.org/10.1097/00005237-199306000-00004

[4] Amanda, N., Taghreed, A. and Seth, A.A. (2014) Neurologic Disease with Pregnan- 
cy and Considerations for the Obstetric Anaesthesiologist. Seminars in Perinatology, 38, 359-369. https://doi.org/10.1053/j.semperi.2014.07.004

[5] Ferrero, S., Pretta, S., Nicoletti, A., Petrera, P. and Ragni, N. (2005) Myasthenia Gravis: Management Issues during Pregnancy. European Journal of Obstetrics \& Gynecology and Reproductive Biology, 121, 129-138. https://doi.org/10.1016/j.ejogrb.2005.01.002

[6] Lu, C.H., Liou, C.M., Chen, Y.S., et al. (1992) Anesthetic Management in Myasthenic Parturient. Anaesthesiologica Sinica, 30, 193-197.

[7] Paterson, I.G., Hood, J.R., Russell, S.H., et al. (1994) Mivacurium in the Myasthenic Patient. British Journal of Anaesthesia, 73, 494-498. https://doi.org/10.1093/bja/73.4.494

[8] Stafford, I. and Dildy, G. (2005) Myasthenia Gravis and Pregnancy. Clinical Obstetrics and Gynecology, 48, 48-56. https://doi.org/10.1097/01.grf.0000153206.85996.07

[9] Chabert, L. and Benhamou, D. (2004) Myasthenia Gravis, Pregnancy and Delivery: A Series of Ten Cases. Annales Françaises d Anesthèsie et de Rèanimation, 23, 459-464. https://doi.org/10.1016/j.annfar.2004.01.014

[10] Fyneface-Ogan, S. and Alagbe-Briggs, O.T. (2013) Anaesthetic Challenges in a High Risk Parturient with Myasthenia Gravis Undergoing Caesarean Section under Spinal Anaesthesia. East African Medical Journal, 90, 338-341.

[11] Quinn, A.C., Milne, D., Columb, M., Gorton, H. and Knight, M. (2012) Failed Tracheal Intubation in Obstetric Anaesthesia: 2 Yr National Case Control Study in the UK. British Journal of Anaesthesia, 110, 74-80. https://doi.org/10.1093/bja/aes320

[12] Eisenkraft, J.B., Book, W.J., Mann, S.M., et al. (1988) Resistance to Succinylcholine in Myasthenia Gravis: A Dose-Response Study. Anesthesiology, 69, 760-762. https://doi.org/10.1097/00000542-198811000-00021

[13] Mushambi, M.C., Kinsella, S.M., Popat, M., et al. (2015) Obstetric Anaesthetists' Association and Difficult Airway Society Guidelines for the Management of Difficult and Failed Tracheal Intubation in Obstetrics. Anaesthesia, 70, 1286-1306. https://doi.org/10.1111/anae.13260

[14] Mir, F., Patel, A., Iqbal, R., Cecconi, M. and Nouraei, S.A.R. (2017) A Randomised Controlled Trial Comparing Transnasal Humidified Rapid Insufflation Ventilatory Exchange (THRIVE) Pre-Oxygenation with Facemask Pre-Oxygenation in Patients Undergoing Rapid Sequence Induction of Anaesthesia. Anaesthesia, 72, 439-443. https://doi.org/10.1111/anae.13799

[15] Rolbin, W.H., Levinson, G., Schneider, S.M. and Wright, R.G. (1978) Anesthetic Considerations for Myasthenia Gravis and Pregnancy. Anesthesia \& Analgesia, 57, 441-447. https://doi.org/10.1213/00000539-197807000-00013 